\title{
Research on Consumer Behavior Characteristics of Shawan Town Based on Sensory Marketing
}

\author{
Yiwen Cui \\ School of Management, Guangzhou College \\ South China University of Technology \\ Guangzhou, China
}

\author{
Qiuling Zhong \\ School of Management, Guangzhou College \\ South China University of Technology \\ Guangzhou, China
}

\begin{abstract}
In 2018, the State Council's government work report proposed that a tourism demonstration area should be created so that tourism and urbanization can develop each other, to look for a "four-in-one" tourism development model for famous site, famous cities, famous towns and famous villages with the help of resources such as famous tourist scenic areas, famous historical and cultural cities and towns and villages, characteristic tourist ancient towns and ancient villages. This article takes consumers in Shawan Town as the research object, and adopts questionnaire surveys and field surveys to investigate consumers' image recognition and behavior characteristics of Shawan Town, through exploratory factor analysis, consumers' five-dimensional influence factors of sight, hearing, touch, taste, and smell in the Shawan Town were refined, and put forward suggestions for improvement from these five dimensions, to help Shawan Town better build a famous historical and cultural town.
\end{abstract}

Keywords-Shawan Town; consumer behavior; sensory marketing

\section{INTRODUCTION}

\section{A. Research Background}

In 2017, "Tourism +" was first written in No. 1 Central Document. Among them, No. 1 Central Document mentioned that it is necessary to cultivate characteristic villages and towns suitable for living and employment, and to create characteristic villages and towns where the primary, secondary and tertiary industries can be integrated and developed. Similarly, the state also supports the development and constructions of major characteristic villages and towns in infrastructure, public services, and a good environment. In 2018, the State Council's government work report proposed that a tourism demonstration area should be created so that tourism and urbanization can develop each other, to look for a "four-in-one" tourism development model for famous site, famous cities, famous towns and famous villages with the help of resources such as famous tourist scenic areas, famous historical and cultural cities and towns and villages, characteristic tourist ancient towns and ancient villages.

\section{B. Research Significance}

At present, although Shawan Town has been appraised as a national AAAA tourist attraction, its tourism development is still not perfect, and there are still many problems that need to be solved and improved. Through an in-depth analysis of the consumer behavior characteristics of Shawan Town based on sensory marketing, not only can it well understand the consumer needs of Shawan Town, but it can also shape the image of Shawan Town in the minds of consumers. In this way, the image of Shawan Town can be more vivid, and the awareness and reputation of Shawan Town can be further enhanced, so that the tourism industry of Shawan Town can develop healthily. The research results can also provide reference ideas and methods for the study of the behavior characteristics of tourists in related ancient towns in China.

\section{MAIN RESEARCH CONTENT}

There are four aspects of Shawan Town were studied from the perspective of consumer behavior. First, through the analysis of tourism status of Shawan Town, this article outlines its tourism development positioning and existing problems. Second, based on the consumer's sensory experience in Shawan Town, this article obtains consumers' information about Shawan Town through field surveys and questionnaires, and then uses sensory experience (visual, hearing, touch, taste, smell) to analyze problems in the Shawan Town. Third, this article uses validity and reliability analysis, exploratory factor analysis and other methods to extract the influencing factors of the visual experience scale, auditory experience scale, tactile experience scale, taste experience scale and tactile experience scale. Fourth, based on the aforementioned survey results of consumer sensory experience, this article proposes solutions for Shawan Town in terms of sight, hearing, smell, taste, and touch, and can also provide some more constructive help for the development of other ancient towns.

\section{A. Current Tourism Status of Shawan Town}

1) Introduction to Shawan Town and its tourism resources

Shawan Town is located in the southwest of Panyu District, Guangzhou, and it is in the middle of the Zhujiang Dlta Region. After more than 800 years of historical development, Shawan Town has gradually formed and retained the south of the Five Ridges culture, which is characterized by water and folk culture. It also represents the Cantonese culture centered on the Zhujiang Dlta Region. 
art have not yet been recognized by consumers. There are also the following problems: the rate of revisiting is not high, the length of stay is different, and it is suitable for 2 people traveling with friends, classmates, colleagues, travel seasons have a large difference, the per capita consumption is low, and most of the consumption items stay in catering, and the travel information is relatively single, the impression of the pavilion stays in Liugengtang, folk arts such as the Shawan fish-lantern museum have received less attention.

At the same time, this article analyzes the sensory experience of consumers in the ancient town of Shawan. In terms of visual experience, buildings such as ancient buildings and ancient ancestral halls are the biggest visual experience for consumers. But Shawan Town has less interaction with consumers in folk art culture such as orchid, Piao-se, fish-lantern, loong dance and lion dance. In terms of auditory experience, consumers enjoy the natural sound in Shawan Town, but consumers have insufficient musical experience with authentic Cantonese music, private group performances, and He's elegant school. In terms of tactile experience, the oyster shell wall is the biggest tactile experience for consumers, but the temperature and humidity of cultural and creative arts and crafts, tables and chairs for visitors to rest and the lounge area are not so obvious. On the sense of taste, Shawan Ginger milk is the deepest taste experience experienced by consumers, but traditional family dishes, specialty foods, morning tea culture and food festival activities are not obvious. In terms of olfactory sensory experience, Shawan Town has not been able to meet consumers' needs for orchids and other flower fragrances.

\section{Scale Analysis of Consumer Sensory Experience}

\section{1) Reliability test}

The overall reliability coefficients of the visual experience scale, the auditory experience scale, the tactile experience scale, the taste experience scale, and the tactile experience scale were $0.901,0.896,0.897,0.898$, and 0.865 , which were greater than 0.7 . Therefore, the overall reliability level of these five scales is high, and the reliability of the questionnaire scales is strong. ("Table I") understanding of consumers' image perception of Shawan Town based on the five angles of sight, hearing, touch, taste and smell.

This survey mainly takes the form of field surveys and interviews in Shawan Town. A total of 168 questionnaires were sent out and 162 questionnaires were recovered, of which 162 were valid questionnaires, with an effective rate of $96.4 \%$. The sample was filled by consumers who have been to Shawan Town. The distribution of the sample is reasonable to represent the population.

\section{2) Descriptive statistical analysis of consumer behavior} in Shawan Town

By studying consumers' impressions of Shawan Town, travel modes, transportation modes, travel seasons, number of trips, and length of stay, per capita consumption and consumption items, ways to obtain travel information, and impressive contents of the pavilion or attractions, this article Discovered that consumers' impression of Shawan Town only stayed in the ancient architectural art. Folk culture and
TABLE I. RELIABILITY STATISTIC SCALES OF CONSUMER SENSORY EXPERIENCE

\begin{tabular}{l|c|c|c}
\hline \multicolumn{1}{c|}{ Content of Scales } & $\begin{array}{c}\text { Number } \\
\text { of Items }\end{array}$ & \multicolumn{2}{|c}{ Cronbach's Alpha } \\
\cline { 1 - 3 } Visual experience scale & 8 & 0.901 & \\
\hline \begin{tabular}{l|c|} 
Auditory experience \\
scale
\end{tabular} & 5 & 0.896 & \multirow{2}{*}{ Reference: } \\
Tactile experience scale & 6 & 0.897 & $\begin{array}{l}\text { Cronbach's } \\
\text { Alpha>0.7 }\end{array}$ \\
\hline Taste experience scale & 6 & 0.898 & \\
\hline Tactile experience scale & 5 & 0.865 & \\
\hline
\end{tabular}




\section{2) Validity test}

The KMO values of the visual experience scale, the auditory experience scale, the tactile experience scale, the taste experience scale, and the tactile experience scale were $0.881,0.833,0.849,0.861$, and 0.805 , which were larger than the common standard of 0.6. And the P values (Sig) corresponding to the Bartlett spherical test are all 0.000 , which are less than 0.05 , which indicates that these data passed the Bartlett spherical test and have a high correlation. The above data indicate that this scale is suitable for exploratory factor analysis. ("Table II")

TABLE II. KMO FOR CONSUMER SENSORY EXPERIENCE AND BARTLETT TEST

\begin{tabular}{l|l|l|l}
\hline \multicolumn{1}{c|}{ Content of Scales } & $\begin{array}{l}\text { Number } \\
\text { of Items }\end{array}$ & $\begin{array}{c}\text { KMO } \\
\text { Value }\end{array}$ & $\begin{array}{c}\text { P-value (Sig) } \\
\text { Corresponding to Bartlett } \\
\text { Spherical Test }\end{array}$ \\
\hline Visual experience scale & 8 & 0.881 & 0.000 \\
\hline Auditory experience scale & 5 & 0.833 & 0.000 \\
\hline Tactile experience scale & 6 & 0.849 & 0.000 \\
\hline Taste experience scale & 6 & 0.861 & 0.000 \\
\hline Tactile experience scale & 5 & 0.805 & 0.000 \\
\hline
\end{tabular}

\section{3) Analysis of exploratory factors}

Through the analysis of exploratory factors, this article has refined the five dimensions of the consumer's vision, hearing, touch, taste, and smell in the Shawan Town. The visual experience is mainly affected by the four aspects of folk art, seasonal feature activities, public facilities, and ancient architecture. The auditory experience is influenced by the two dimensions of Cantonese music and ambient sound. The tactile experience is influenced by the three aspects of humanistic art touch, public facilities touch and plant touch. Taste experience is influenced by three aspects: traditional cuisine, gourmet activities, and Shawan Ginger milk. The olfactory experience is noticed in three areas: the smell of the public area, the smell of flowers, and the smell of food. Therefore, Shawan Town can design marketing strategies from the above aspects to enhance consumers' perception of the ancient town and enhance the brand image of Shawan Town. ("Table III")

TABLE III. INFLUENTIAL FACTORS OF SENSORY DIMENSIONS

\begin{tabular}{l|l}
\hline \multicolumn{1}{c|}{ Dimensions } & \multicolumn{1}{c}{ Influential Factors } \\
\hline \multirow{4}{*}{ Vision } & Folk art \\
\cline { 2 - 2 } & Seasonal feature activities \\
\cline { 2 - 2 } & Public facilities \\
\cline { 2 - 2 } Hearing & Ancient architecture \\
\cline { 2 - 2 } Touch & Cantonese music \\
\hline \multirow{2}{*}{ Taste } & Humbient sound \\
\cline { 2 - 2 } & Public facilities touch \\
\hline & Plant touch \\
\hline & Traditional cuisine \\
\hline & Gourmet activities \\
\hline Smell & Shawan Ginger milk \\
\hline & The smell of the public area \\
\hline
\end{tabular}

Source of data: Organized by the author

\section{MARKETING SUGGESTIONS BASED ON CONSUMER SENSORY EXPERIENCE}

\section{A. Suggestions on Brand Visual Experience}

1) Carrying out the activities of appreciating ancient architecture

Although the ancient architectural art is difficult to imitate and change again, it is possible to let consumers know the historical origin and story development of the ancient architecture through the appreciation of the ancient architectural art. This can enhance the charm of consumers to experience the ancient architectural art in Shawan Town, and have a deeper understanding of the ancient architectural art in Shawan Town.

\section{2) Experiencing folk art}

The successors of Shawan fish-lantern hall and Shawan ancient decoration exhibition hall are invited to let the inheritors display and explain the works in Qingshuijing Square and Sifangtang Square. When necessary, Shawan Town can also invite Shawan Town consumers to come and try to make their own fish-lantern or sculpture works. Shawan Town can receive free activities for making fish lanterns and free carving works by forwarding the circle of friends for free, which can attract consumers to sign up. In Liugengtang, Shawan Piao-se inheritor was invited to explain the floating color palette, so that consumers of parent-child travel can experience the art of Piao-se little people properly.

3) Increasing the exposure of loong dance and lion dance and orchids

The first is the successor of loong dance and lion dance. In Tik Tok, the "Who is the real successor of loong dance and lion dance" event was invited to invite Shakeng's successor of loong dance and lion dances to take a video with their students. In this way, the online publicity activities can be diverted to the offline, and then the loong dance and lion dance exchange activities will be carried out in Shawan Town, so that consumers playing in Shawan Town can also feel the dragon and lion culture. The second activity is to 
carrying out experiential activities in making orchid specimens.

4) Creating cultural and creative products with brand visual personality

Cultural and creative products can better convey seasonal cultural characteristics. According to the previous analysis, due to the strong seasonality of the special events in Shawan Town, the true features of Shawan Town are not well recognized by consumers. Therefore, Shawan Town can start from cultural and creative products, enhance tourists' visual experience of the ancient town, and increase income while disseminating brand positioning.

In the design of cultural and creative products, the ancient town can make fish-lantern earrings, make postcards of ancient buildings, make interesting personal animation sculptures, shoot tumbler toys with Sezai figures in Piao-se, and make scarves and jewelry with orchids.

\section{B. Suggestions on Brand Hearing Experience}

1) Strengthening the dissemination of Cantonese music

First, increase the number of private group performances. Second, increase the number of lecturers at Cantonese Music Hall in Shawan. Third, open an official account on the Himalayan platform and recording professional musicians explaining Cantonese music on the Himalayan platform, so that users of the Himalayan platform can also recognize the position of Shawan Town as the hometown of Cantonese music. Fourth, invite well-known music writers to endorse, making Shawan Town's positioning and publicity as the hometown of Cantonese music more obvious.

\section{2) Improving environmental sound}

First is to create a natural sound. According to the survey, consumers pay more attention to natural sound. Therefore, Shawan Town raises birds, such as sparrows, parrots and pigeons, making Shawan Town be full of life. Shawan Town can also use the wind bell to strengthen the consumer's imprint on Shawan Town. By attaching a wind chime on the fish-lantern and using the power of natural sound, the wind in Shawan Town will be blown, letting a pleasant sound entangle in the ears of consumers.

Second, there is no interpreter in the exhibition hall of Shawan Town. Therefore, Shawan Town can walk into the campus and sponsor students' activities to attract students with "find the most unique features of Sandy Bay" as the competition activity, and those who perform well can get summer vacation intern.

\section{Suggestions on Brand Tactile Experience}

1) Opening a store of cultural and creative products

In the design of cultural and creative products, Shawan Town can cooperate with Guangzhou Academy of Fine Arts in Panyu university town to host a cultural and creative product design competition in Shawan Town, allowing students to design cultural and creative products with Shawan characteristics, then Recycle and use excellent cultural and creative products designed by students.

\section{2) Increasing public rest areas}

Shawan Town can add public rest areas, such as the tables and chairs for rest, near the Academician He Binglin Memorial Hall, on the first floor of Sifangtang and the Ancient Decorative Arts Museum. Tables and chairs are made of wood and steel. It is best to arrange them in a row. This will not take up much space and allow consumers to sit down.

\section{3) Establishing plant corridors}

Shawan Town can attract consumers by establishing a plant promenade to add tactile experiences in summer and autumn. A plant promenade is set up in the street between Yiwutang and Shawan Dairy Cow Queen. Hanging above the plant promenade is wood, and then planting vines and flowers to grow along the wood. The main types of plants are orchids, supplemented by vines, so that consumers can take advantage of the hot summer.

\section{Suggestions on Brand Taste Experience}

\section{1) Strengthening the taste experience of Shawan Ginger} milk

Shawan Town can shoot the story behind Shawan Ginger milk and upload it to Shawan Town's official Tik Tok. The content of the story is that an old woman in Shawan Town had a cough disease, but it was a coincidence that she poured ginger juice with water and milk and the milk solidified. Later the old woman drank it and got better. After the He family knew this, they asked the grandmother how to make it. Later, relatives of the He family used this method to make desserts in Shawan Town.

\section{2) Carrying out tasting activities of Shawan cuisine}

According to the analysis of the previous content, in addition to Shawan Ginger milk, other cuisines such as traditional soups and specialties are less well known. Therefore, Shawan Town and other merchants can carry out Shawan Food Tasting activities so that consumers can experience the taste of Shawan Town from the taste.

\section{3) Finding the strongest foodie in Shawan}

Looking for the strongest foodie in Shawan cuisine and acting as an opinion leader, then consumers can quickly find the traditional local cuisine in Shawan Town.

4) Inviting We-Media to find daintiness in Shawan Town

Gourmet We-Media has a strong communication ability, which can make more consumers understand the food in Shawan Town. Shawan Town invites the well-known gourmet public number in Guangzhou to taste free food in Shawan Town. Such as Guangzhou eating and drinking, eating and drinking IN Guangzhou, Guangzhou food circle, SOHO Guangzhou, talking and eating, Guangzhou food group, Guangzhou snacks light, eating and drinking Zengcheng, Guangzhou food strategy. Shawan Town let them evaluate the freshness, taste, price, and serving size of each Shawan traditional cuisine. We-Media has selected the most cost-effective traditional cuisine for each cuisine. Gourmet We-Media personally experienced the production process, and presented the process of personal experience to 
habit of parading more than two people. The non-spring attraction is insufficient, and the number of trips and stay time are polarized. They have low per capita consumption and single consumption items, and pay attention to behavioral characteristics such as recommendations from friends and relatives.

Through exploratory factor analysis, this article finds that in terms of consumer visual experience, four factors, namely folk art, seasonal Shawan cultural characteristics, public facilities, and ancient architectural art, have been refined. In terms of auditory experience, two influence factors, Cantonese music and ambient sound, have been extracted. In terms of tactile experience, three influencing factors of human art touch, public facilities touch and plant touch have been extracted. In terms of taste experience, three influence factors can be extracted: traditional food, food activities and Shawan Ginger milk. In terms of olfactory experience, three factors affecting the public area fragrance, flower fragrance and food fragrance were extracted.

According to the extracted impact factors, this article puts forward five suggestions from the perspective of sensory marketing. In terms of visual experience, Shawan Town interacts with consumers by appreciating ancient architectural activities, experiencing folk art, and increasing the exposure of loong dance and lion dance and orchids. In terms of auditory experience, private group performances, interpretation of He's elegant music, registration of Himalayan account and other ways to strengthen consumers' awareness of the hometown of Cantonese music. In terms of haptics, visitors will be able to improve their tactile experience in Shawan Town by opening stores for cultural and creative products, increasing tables and chairs in public rest areas, adjusting the temperature and humidity in the exhibition hall, and establishing plant corridors. In terms of taste, the use of enhanced taste of Shawan Ginger milk, free tasting of Shawan cuisine and finding the strongest foodie of Shawan cuisine will make consumers feel the taste of Shawan cuisine on their tongues. In terms of smell, through the exhibition of orchids and increasing the coverage of orchids in different months, consumers' awareness of China's famous orchid towns has been increased. orchid town in China from its sense of smell. Through the photography and dissemination of professional photographers, the image of the famous Chinese orchid town can be implemented in the hearts of consumers.

\section{CONCLUSION}

After more than 4 years of operation and development in Shawan Town, the biggest features that consumers experience in Shawan Town are ancient architecture, natural sound, oyster shell walls, Shawan Ginger milk and fresh air. This does not match the positioning of Shawan Town as the hometown of Chinese sculpture, the hometown of Chinese fish-lantern, the hometown of Chinese loong dance and lion dance, the hometown of Cantonese music, the famous Chinese orchid town, and the characteristics of Shawan itself.

For Shawan Town, consumers pay more attention to unique architectural styles of Liugengtang Pavilion, as well as the Lingnan culture and folk art culture. They show the

\section{REFERENCES}

[1] Zhong Ke, Wang Haizhong, Yang Chen. Review and Prospect of Sensory Marketing Research [J]. Foreign Economics \& Management, 2016, 38 (05): 69-85 (in Chinese)

[2] $\mathrm{Xu}$ Ming. Application of sensory marketing strategy and cultural marketing strategy in hotel industry [J]. China Market, 2009 (27): 1213 (in Chinese)

[3] Liao Bin. The application of sensory marketing strategies in the guesthouse industry [J]. Tourism Overview (Second Volume), 2018 (04): 54 (in Chinese)

[4] Liu Wumei, Wang Haizhong, Wang Jingyi. Tactile Research in the Field of Consumer Behavior: Review, Application and Prospects [J]. Foreign Economy \& Management, 2014, 36 (04): 25-35 (in Chinese)

[5] Gao Fang. Review and Prospect of the Research on the Effect of Sensory Stimulation on Consumer Behavior [J]. Journal of Brand Research, 2016 (02): 63-70 (in Chinese) 